\title{
Next Generation of Energy Residential Gateways for Demand Response and Dynamic Pricing
}

\author{
A. CUEVAS, C. LASTRES, J. CAFFAREL, R. MARTÍNEZ, A. SANTAMARÍA, Member, IEEE \\ Centro de Domótica Integral (CeDInt-UPM), Universidad Politécnica de Madrid. Spain
}

\begin{abstract}
Predictions about electric energy needs, based on current electric energy models, forecast that the global energy consumption on Earth for $\mathbf{2 0 5 0}$ will double present rates. Using distributed procedures for control and integration, the expected needs can be halved. Therefore implementation of Smart Grids is necessary. Interaction between final consumers and utilities is a key factor of future Smart Grids. This interaction is aimed to reach efficient and responsible energy consumption. Energy Residential Gateways (ERG) are new in-building devices that will govern the communication between user and utility and will control electric loads. Utilities will offer new services empowering residential customers to lower their electric bill. Some of these services are Smart Metering, Demand Response and Dynamic Pricing. This paper presents a practical development of an ERG for residential buildings.
\end{abstract}

\section{INTRODUCTION}

Technology evolution and continual energy demand growth [1] highlight the need of innovative tools that allow sustainable development of power systems [2]. Smart Grids offer new services such as Demand Response (DR), Dynamic Pricing (DP) and Energy Management (EM) to customers [3].

For efficient power supply distribution new network architectures should be developed, creating a new generation of Smart Grids with new capabilities, such as:

- High degree of interoperability, synchronism and integration.

- Communication with existing devices.

- Active control of energy demand (based on price warnings and demand peaks).

- Management of distributed renewable energy systems.

Among the services provided by Smart Grids, Demand Response (DR) contributes to control the demand in the residential environment as a response to a price signal or a reliability-based action. DR services pursue [4]: Peak clipping (to decrease demand at critical periods); Load Shifting (redistribution of demand based on energy prices) and Valley Filling (increase of demand at low-priced periods). These services are carried out under different pricing policies:

- Dynamic Pricing (DP)[5]: such as Critical Peak Pricing (CPP), Time of Use (ToU) and Real Time Pricing (RTP)[6] programs.

- Demand Adjustments Incentive-based programs: classified as remote control and non remote control.

This paper presents a practical development of an ERG for

Authors would like to thank CDTI (Centro para el Desarrollo Tecnológico Industrial) of Spain for the support provided through the funded project DENISE (CENIT 2007-1002). residential buildings .This ERG represents an easy to use and flexible solution to bring Smart Grids to the residential environment.

\section{ERG INTERFACE TO THE SMART GRID ARCHITECTURE}

The Energy Residential Gateway here presented has been designed to be part of a new Smart Grid model that includes a Utility Smart Grid Manager (USGM) to manage the distribution of messages among the utility transformer substation and homes, as shown in Fig. 1.

The core of the Smart Grid is the Utility Management Platform (UMP), which allows the interconnection of network elements, processes and services covering distributed generation systems, utility power supply and final user devices.

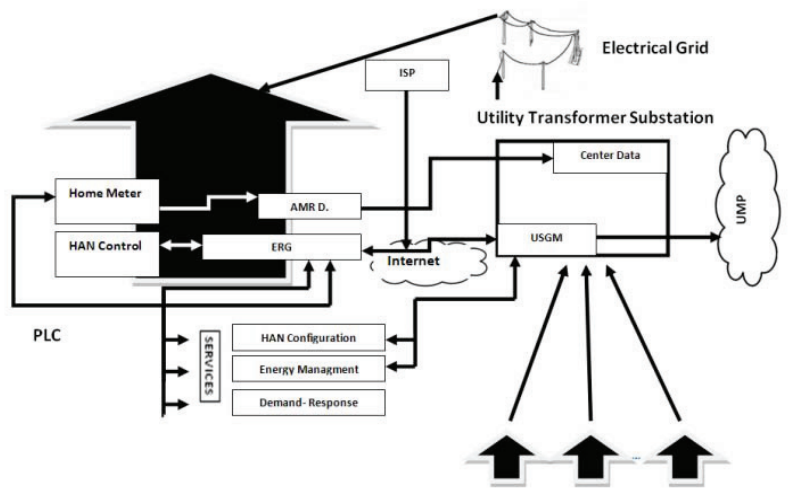

Fig. 1-Smart Grid Architecture - Interconnection with ERG

This architecture pursues the following goals:

- Home Energy Consumption: monitoring, data logging and processing.

- Loads Management Control with or without human presence at home.

- Monitor eventualities on power supply.

- Bidirectional communication between utilities and homes.

- Smooth Power Demand Tracking.

Communications between USGM and the Energy Residential Gateway are established through an Internet connection provided by an ISP, whereas Power Line Communication (PLC) is used in the Home Area Network (HAN). Within the HAN architecture, the ERG controls home devices and interacts with home meters through X-10 and A10 protocols, based on PLC.

\section{SERVICES ENABLED BY THE ERG}

The requirements of the Energy Residential Gateway have 
been defined to provide new services such as:

\section{A. HAN Configuration and Control Services up to 256 devices.}

\section{B. Energy Management Services}

- Monitor Energy Consumption: shows the household energy consumption.

- Data transfer: the ERG sends periodically the energy consumption to the USGM.

- Users' information services: provide visualization of user's tariff, event logs and alert messages.

\section{Demand Response and Dynamic Pricing Services}

The Energy Residential Gateway offers a set of Demand Response services, focused on peak clipping and valley filling actions, according to the algorithm shown in (Fig. 2).

ERG reacts to emergency DR messages from the Utility, decreasing power demand at critical periods (Service no.1). This message contains the percentage of power reduction required. HAN devices will be re-configured in order to adjust household consumption to this new target.

Regarding Valley Filling, the gateway is ready to switch on or dimmer some HAN devices when a low price RTP message is received from the utility (Service no.2). In both scenarios, the ERG discovers automatically those HAN devices ready to be switched or dimmered.

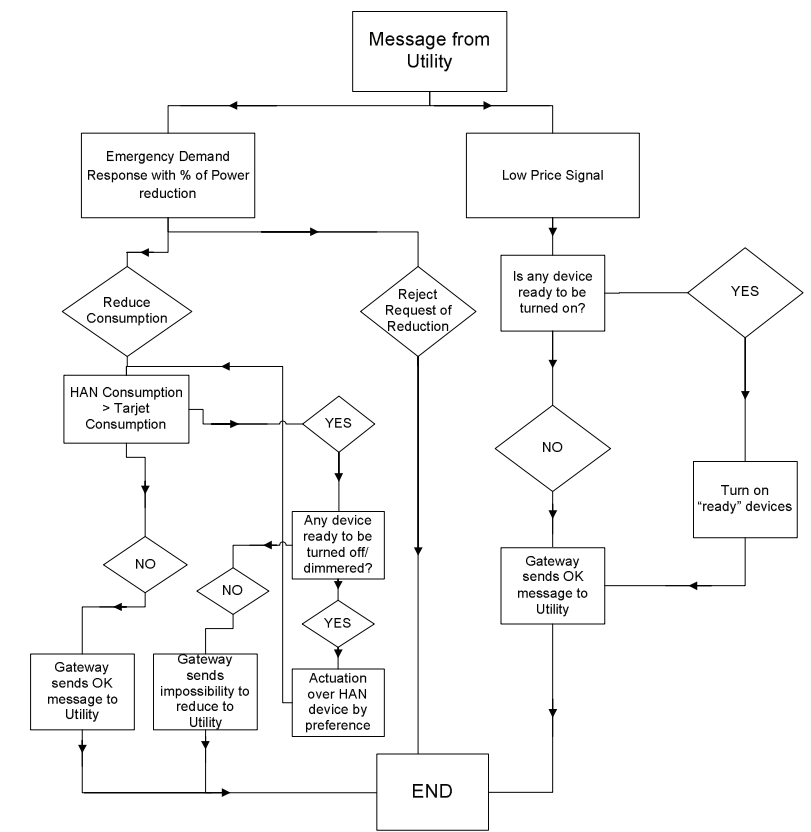

Fig. 2- ERG algorithm for Demand Response and Dynamic Pricing services.

Messages received from the utility have the following structure:

"type of_DRService::hour::date::percentage_of_reduction" e.g. " $1:: 21.59:: 02 / 10 / 2010:: 75.0 "$ ".

\section{BUILDING MODULES OF THE ERG}

ERG has been developed on an ARM926 processor based platform, using a LCD Touchscreen as user interface. Metering and communication functions are performed by two external devices: a Home Meter and a X.10 interface.

The ERG Firmware comprises the following functional modules:

- Control module: sets up the communication with the Home Meter and the HAN (X.10).

- Graphical User interface (GUI) module: based on Qt libraries, consist of graphical elements such as windows, menus, radio buttons and icons that provide users the tools to interact with the ERG. A friendly and intuitive GUI is presented to provide customers with an easy way to control their HAN.

- Energy Management and Demand Response module: provides HAN control, consumption monitoring, scheduling events, electricity agreement details and demand adjustment services focused on peak clipping and valley filling actions.

\section{PRACTICAL IMPLEMENTATION AND CONCLUSIONS}

The Energy Residential Gateway here presented has been successfully installed at a test lab in the Center for Energy Efficiency and Smart Buildings (CeDInt-UPM).

This test lab is equipped with several X-10/A-10 appliance modules to provide control services over different home appliances (washing machine, dishwasher, PC, a group of six lamps, two Venetian blinds, etc.).

After thoroughly testing the ERG, the following results have been obtained: a wide range of HAN devices has been successfully configured; the measured average response time of the tested HAN devices is approximately three seconds; after receiving different messages simulating Demand Response and Dynamic Pricing services, the ERG proceeds with the necessary actions into the HAN in an average time of thirty seconds.

This short reaction time ensures that the ERG contributes to achieve peak clipping whenever any event occurs in the electricity distribution network.

\section{REFERENCES}

[1] [ D. Coll-Mayor, M. Paget, and E. Lightner, "Future intelligent power grids: Analysis of the vision in the European Union and the United States," Energy Police, vol. 35, 2006, pp. 2453-2465.

[2] C. Batle, and P. Rodilla, "Electricity Demand Response Tools: status quo and outstanding issues," European Review of Energy Market, 2008.

[3] C. Su, and D. Kirschen, "Quantifying the Effect of Demand Response on Electricity Markets".IEEE Transaction on Power Systems, vol. 24, no. 3, 2009.

[4] F. Andersen, S. Jensen, H. Larsen, P. Meibon, H. Ryan, K. Skytte, and M. Togeby, "Analysis of Demand- Response in Denmark". Riso Report, 2006.

[5] G. Heffner, "Demand Response Valuation," Global Energy Associates, 2009

[6] FERC, "Assessments of Demand Response and Advanced Metering," Federal Energy Regulatory Commission, 2006. 